\title{
A Novel Approach for Detection of Anomalies in Images related to Diabetic Myonecrosis
}

\author{
Fahimuddin. Shaik ${ }^{1}$, Dr.Anil Kumar Sharma ${ }^{2}$, Dr.Syed. Musthak Ahmed ${ }^{3}$ \\ Research Scholar, Electronics \& Communication Engineering, Sunrise University-Alwar, Rajasthan ${ }^{1}$ \\ Professor \& Principal, Institute of Engineering \& Technology, Alwar, Rajasthan ${ }^{2}$ \\ Professor and HOD, Department of ECE, SREC, Warangal, Telangana State ${ }^{3}$
}

\begin{abstract}
Diabetes mellitus (DM) and its complications is a comprehensive challenge to health care systems. Diabetic Myonecrosis or diabetic muscle infarct (DMI) is a rare complication of diabetes or possibly under diagnosed. Image processing methods have become crucial in solving various medical Imaging problems. The proposed work is mainly dealt with processing of medical images related to Diabetic Myonecrosis. A novel approach is designed in this paper by having Hybrid Morphological Reconstruction (HMR) Technique as Pre-Processing With Watershed Segmentation Method as Post-Processing.
\end{abstract}

Keywords: Image, segmentation, necrosis, morphological, watershed.

\section{INTRODUCTION}

Diabetic mellitus (DM) is a metabolic disorder that order to reduce the influence from undesirable variations characterized by inability of the pancreas to control blood within, the Hybrid Morphological Reconstruction (HMR) glucose concentration. This predicament results may make [12] is used to enhance the image out blood glucose levels out of range [2]. It is described in longstanding diabetes. Although it was first reported in 1965 , only case reports and a systemic review of these cases have been published [3, 4]. Clinical presentation is with acute onset of muscle pain, commonly the thigh. Diabetes Myonecrosis can be misdiagnosed as cellulitis, deep vein thrombosis or facsiatis. Magnetic resonance imaging (MRI) is sufficient, in the appropriate clinical context, to enable the diagnosis, hence avoiding inappropriate treatment [11].

On a Technical note Image Processing is utilised to extract important features from the images, through which better perception of the scene can be obtained for human viewers [1]. The biological vision system is one of the most important means of exploration of the world to humans, making complex task easier for betterment of understanding [7].

There are numerous algorithms that can be utilised for different applications but enhancement and segmentation are considered as most sort out methods for improving the details in an image. It is not possible to judge that any one method is best in Image processing applications but one can use trial and error method as a practical approach for obtaining the perfect results. Image Enhancement is a fundamental task in digital image processing and analysis, aiming to improve the appearance of image in terms of human brightness perception [8]. Whereas the Segmentation is mainly useful in classification of objects and labelling of the features extracted from image for easy analysis. One should look into that processing of images is done without blemishing the integrity of original image.

\section{HYBRID MORPHOLOGICAL RECONSTRUCTION}

Due to the imperfection and variations, the appearance of microscopic images is generally not homogeneous. In

\section{WATERSHED SEGMENTATION}

Watershed segmentation falls under Morphological image processing methods and is a distinguished image segmentation technique because of its significance related to mathematical morphology. Morphological operators have been applied for vasculature segmentation [9] because the fundamental morphology of the vasculature is known a priori to be comprised of linked linear segments and because of speed and noise resistance. The concept of watersheds is based on visualizing an image in 3 dimensions given by two spatial co-ordinates versus intensity. Here one can consider only three points for clear explanation of the topic and they are (a) points belonging to regional minimum (b) points at which a drop of water, if placed at the location of any of those points, would fall with indeed to a single minimum; and (c) points at which water would be equally likely to fall to more than one such minimum. The points fulfilling condition (c) form crest lines on the topographic surface and are termed divide lines or watershed lines [6].

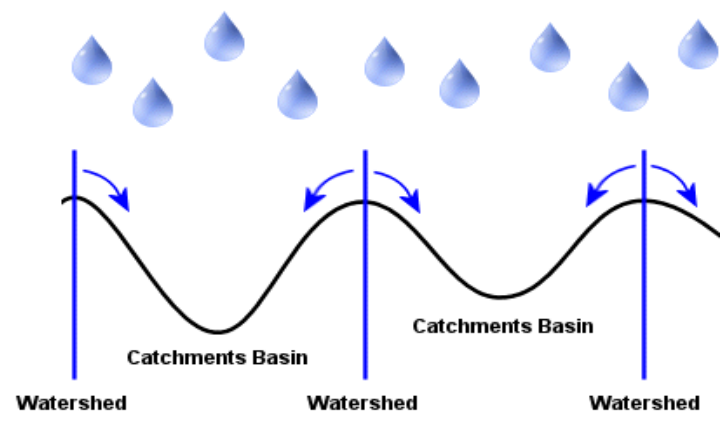

Fig 1. Watershed representation

The standard objective of this method is based on the concept to find watershed lines. The basic idea is simple, 
suppose that a hole is punched in each regional minimum and that the entire geography is flooded from below by allowing water rise through the holes at uniform rate [5]. The entire process is described by a concept that a dam like thing is constructed to avoid merging and flooding may take place when water reaches the top level of dam. Consequently, watershed algorithm extracts the boundaries. In [10] watershed algorithm was used for segmentation of splats, a collection of pixels with similar color and spatial location.

\section{EXISTING WORK}

In literature $[11,12]$ the above said algorithms have been used as pre-processing or post-processing methods with other algorithms. But in this work these two algorithms are combined to form a hybrid model to attain effective results for easy classification and analysis of the inner lying cause of the anomalies present in the images related to Diabetes.

\section{PROPOSED WORK}

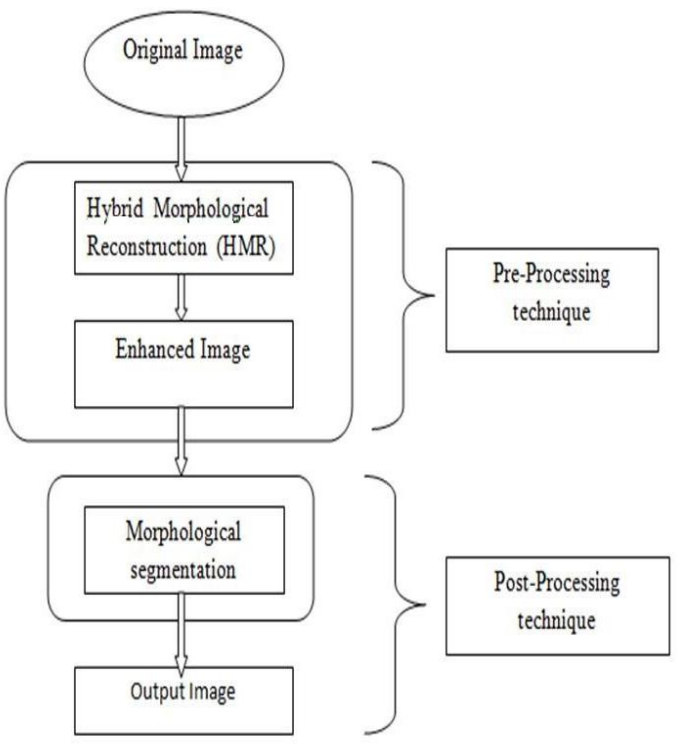

Fig 2. Block Diagram of Implemented Model

The implemented method is used to enhance and segment the medical image. Here Hybrid Morphological Reconstruction (HMR) technique is used to enhance the medical images, and Morphological segmentation is the enhanced medical image.

\section{Model description:}

The images that are obtained from public database Image Courtesy: Radiology and Molecular Imaging; Sultan Qaboos University Hospital, Al Khod, Oman. Hence the image is processed using the MATLAB software and the image undergoes several algorithms to get a better output. Initially the RGB image is converted into grey scale to avoid complex calculations. Next step is to perform the Gradient Magnitude segmentation function. After the above two steps are finished then the main step, watershed transform segmentation is performed. Watershed transform is the region base segmentation method. In this step it fills the gaps present in the images and finally the analyzing the result.

\section{RESULT AND ANALYSIS}

The original image (Figure 3) considered in this work is an axial fat-suppressed T2-weighted image which shows enlargement and increased signal intensity of the vastus lateralis muscle $(*)$ and rectus femoris (open white arrow). There is fluid in subcutaneous tissue and intermuscular fascia (black arrow).

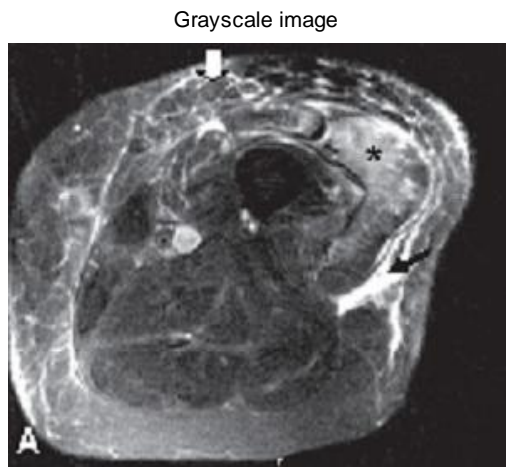

Fig 3. Axial fat-suppressed T2-weighted image (Image Courtesy: Radiology and Molecular Imaging ;Sultan Qaboos University Hospital, Al Khod, Oman.)

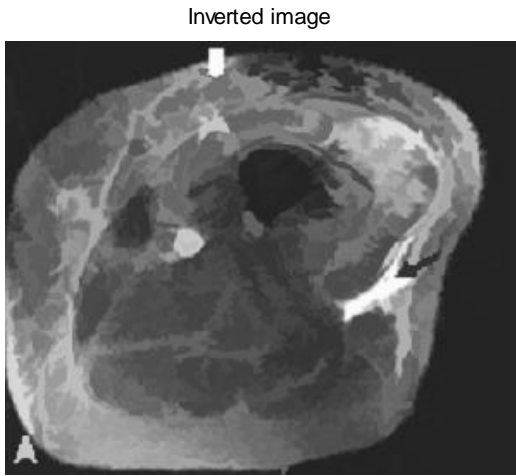

Fig 4.Inverted Image

Gradient magnitude (gradmag)

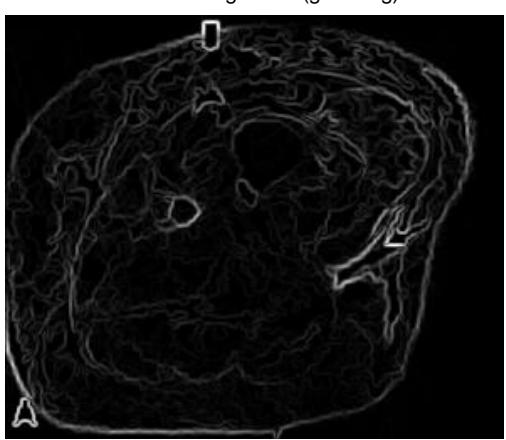

Fig 5.Gradient Magnitude Image

The result obtained in figure 4. shows the enhancement of vastus lateralis muscle and intermuscular fascia areas of original image. And after application of Gradient magnitude method of post-processing results in clear threshold image (Figure 5) of intermuscular fascia part of the original image. Whereas in reconstruction process from figure 6 it is evident how intermuscular fascia part enlarges and may further result in necrosis of the muscle leading to permanent damage of the muscle shown with Gray shaded area which is represented by black arrow. 
INTERNATIONAL JOURNAL OF INNOVATIVE RESEARCH IN ELECTRICAL, ELECTRONICS, INSTRUMENTATION AND CONTROL ENGINEERING Vol. 3, Issue 12, December 2015

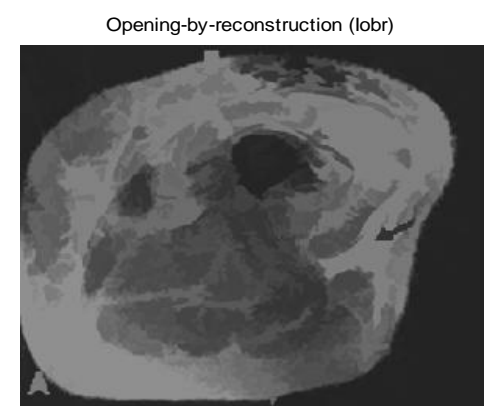

Opening-closing by reconstruction (lobrcbr)

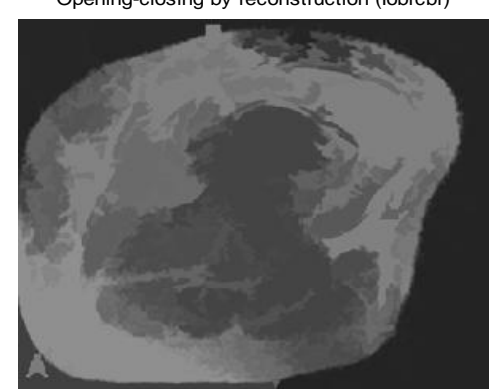

Fig 6. Reconstruction process suggestive of muscle necrosis with different gay shades (indicating muscle can be cut down into fibrous parts).

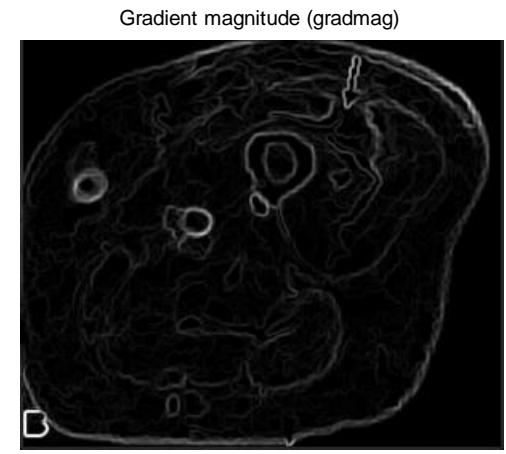

Fig 9 Gradient Magnitude

And after application of Gradient magnitude method of post-processing results in clear empty area formed in vastus lateralis (Figure 9) due to lack of healthcare towards Diabetes Mellitus.

\section{CONCLUSION}

The experimental results of Hybrid Morphological Reconstruction Technique as Pre-Processing with Watershed Segmentation Method as Post-Processing are quite suitable for educating the common man related to Diabetic Myonecrosis. In future development other preprocessing algorithms combined with the implemented post processing method can give perspective results so that the Medical Professionals may make use of this algorithm for earlier detection of the abnormality and this framework in form of a group wise images may be used to educate a common about seriousness of the problem.

\section{ACKNOWLEDGEMENT}

The authors are thankful to Sunrise University-Alwar, Rajasthan and Annamacharya Institute of Technology \& Sciences, Rajampet, A.P. for providing research facilities. And also thankful to Dr.B.Jayabhaskar Rao, Diabetalogist, Diabetic Care Center Nandalur, A.P. for providing the detailed explanation of Diabetes and its abnormalities.

\section{REFERENCES}

[1] Rafael C. Gonzalez, Richard E.woods "Digital Image Processing”, ADDISON-WESLEY. An imprint of Pearson Education, 1st edition

[2] Sharifi, A., Vosolipour, A., Aliyari Sh, M.; Teshnehlab, M "Hierarchical Takagi-Sugeno Type Fuzzy System For Diabetes Mellitus Forecasting" proc. Of 7th Int. Conf. on Machine Learning and Cybernetics, Kunming, Vol 3,pp.1265 - 1270, 12-15 July 2008

[3] Angervall L, Stener B. Tumor form focal muscular degeneration in two diabetic patients. Diabetologia1965; 1:39-42 .10.1007/ BF0 1338714

[4] Trujillo-Santos AJ. Diabetic muscle infarction: an under diagnosed complication of long-standing diabetes. Diabetes Care 2003. Jan;26 (1):211-215 10.2337/diacare.26.1.211

[5] R.Ravindraiah, Fahimuddin.Shaik, "Detection of Exudates in Diabetic Retinopathy images\| National conference on "Future Challenges and Building Intelligent Techniques in Electrical and Electronics Engineering "(NCEEE" 10),pp 363-368,Chennai, INDIA, July 2010.

[6] Fahimuddin.Shaik, Dr.M.N.Giriprasad, C.Swathi, A.Soma Sekhar, -Detection Of Cardiac Complications In Diabetic Patients Using Clahe Method\| Proceedings Of International Conference On Aerospace Electronics, Communications And Instrumentation(Aseci-2010), Pp 344-347.6-7 ,India Jan 2010. 
[7] F.A.Peres, F.R.Oliveira, L.A.neves, M.F.Godoy "Automatic Segmentation of Digital Images Applied in Cardiac Medical Images" IEEE -PACHE, Conference, Workshops, and Exhibits Cooperation, Lima, PERU, March 15-19, 2010.

[8] Intajag, S.; Tipsuwanporn, V.; Chatthai, R Chatree "Retinal Image Enhancement In Multi-Mode Histogram” 2009 World Congress on Computer Science and Information Engineering, Vol 4, pp. 745749, Mar 2009

[9] F. Zana and J.-C. Klein, "A multimodal registration algorithm of eye fundus images using vessels detection and hough transform," Medical Imaging, IEEE Transactions on, vol. 18, no. 5, pp. 419428, 1999.

[10] L. Tang, M. Niemeijer, and M. D. Abramoff, "Splat feature classification: Detection of the presence of large retinal haemorrhages," in Biomedical Imaging: From Nano to Macro, 2011 IEEE International Symposium on. IEEE, 2011, pp. 681-684.

[11] Kattapuram TM, Suri R, Rosol MS, Rosenberg AE, Kattapuram SV. Idiopathic and diabetic skeletal muscle necrosis: evaluation by magnetic resonance imaging. Skeletal Radiol 2005. Apr;34(4):203209 10.1007/s00256-004-0881-8

[12] Amit Kumar, Fahimuddin.Shaik, "Image Processing in Diabetic Related Causes”, Springer-Verlag Singapur Publishers (Springer Briefs in Applied Sciences and Technology-Forensics and Medical Bio-informatics), May 2015. (ISBN: 978-981-287-623-2) (http://www.springer.com/in/book/9789812876232\#aboutBook )

[13] Cheng Lu, Muhammad Mahmood, Naresh Jha, Mrinal Mandal. "A Robust Automatic Nuclei Segmentation Technique for Quantitative Histopathological Image Analysis ", Analytical and Quantitative Cytology and Histopathology. December 2012, P. 296-308

\section{BIOGRAPHIES}

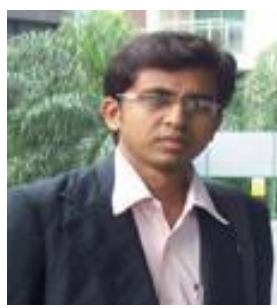

Fahimuddin. Shaik received B.Tech \& M.Tech Degrees in Electronics \& Communication Engg. from JNT University, Hyderabad, India. He is currently working towards $\mathrm{PhD}$ Degree in Biomedical Image Processing at Sunrise University, Alwar, Rajasthan, India. He is with Annamacharya Institute of Technology \& Sciences, Rajampet, A.P., India working as an Assistant Professor in Dept. of ECE. He is BOS Member of the Department and also held a position as the Academic Council Member of the Institute. He is a member of Professional societies like IEEE, ISTE (India), BMESI (India), IACSIT (Singapore), IAENG (Hongkong) and WASE (Hongkong). His research interests include Signal Processing, Time Series Analysis and Biomedical Image Processing. He chaired a Session at IEEE International Conference (ICMET-2010) held in Singapore on Sep' $11^{\text {th }} 2010$ and also in ICCET-12 in 2012 at Kollam, Kerala. He was Scientific Committee member of III Workshop on Technology for Healthcare and Healthy Lifestyle, 2011(WTHS ${ }^{\text {ee }}$ 11), Valencia, Spain, 1st-2nd December 2011. He has authored books by titles "Medical Imaging in Diabetes" with Cinnamonteal Publishers in 2011 and "Image Processing in Diabetic Related Causes" with Springer Publications in 2015.

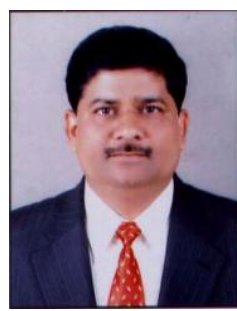

Dr.Anil Kumar Sharma received his M.E. degree in Electronics and Communication Engineering from Birla Institute of Technology, Deemed University, Mesra, Ranchi and $\mathrm{PhD}$ from Singhania University, Rajasthan. He has an experience of 20 years on various RADARs and Communication
Equipments and 9 years of teaching experience. He is currently Principal of Institute of Engineering and Technology, Alwar, Rajasthan, India. He has published technical papers in International journals as well as in international and national conferences. He is Reviewer and Editor of reputed Journals. He is a member of Professional societies like ISTE (India), IACSIT (Singapore), IAENG (Hongkong) and. His research and teaching interest include Microprocessor, VLSI Design, Neuro-Fuzzy Modeling, RADARs \& its Data Handling Systems. He has attended four days study and Networking Tour at UTP, Malaysia to visit Laboratories and R\&D Facilities in the areas of Intelligent Signal \& Imaging and Nanotechnology in 2014. He has authored books by titles "Digital Logic Design and "Basic Electrical and Electronics Engineering". At present he is guiding $\mathrm{PhD}$ Research Scholars at RTU, Kota and SRU, Alwar.

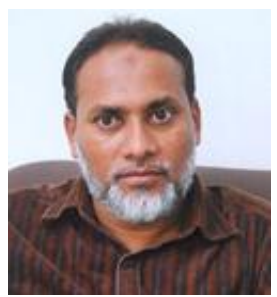

Dr. Syed. Musthak Ahmed received his B.E. (Electronics), M.E. (Electronics) degrees in 1986 and 1998 respectively from Bangalore University and received $\mathrm{PhD}$ from Vinayaka Missions University, Salem in 2011. He worked as Asst. Professor and Head of the Department (ECE) at Ghousia College of Engineering of Vishweshwaraiah Technological University for 20 years and has been a senior Associate Professor in Jawaharlal Technological University since 2007 . His research interest includes MEMS Applications, IC fabrication, Control systems etc. He has 28 years of teaching experience in reputed engineering colleges and he is presently working as Prof \&HOD (ECE), SR Engineering College, Warangal. He is Doctoral committee member as well as Indian examiner in reputed universities. He was invited as a special invitee for BoS, of PG (EDT) program by the affiliating University JNTUH. He is Member \& Convener of Research Committee of SREC, Warangal. He was recognized as one of the Evaluators for National Board of Accreditation, New Delhi. He is Vice Chairman, IEEE Education Society Chapter, IEEE Hyderabad Section for the academic year 2015.He was Co-chair for SIESCON11 conference, Dr. M.G.R Anna University, Chennai. He is a member of ISTE, IEEE, FISSS, and FIETE. He has various publications in National \& International Journal/Conferences. 